\title{
Adult-onset multiple mitochondrial DNA deletion syndrome due to DGUOK deficiency
}

INSERM

\section{Source}

INSERM. (1999). Orphanet: an online rare disease and orphan drug data base. Adultonset multiple mitochondrial DNA deletion syndrome due to DGUOK deficiency. ORPHA:329314

Adult-onset multiple mitochondrial DNA deletion syndrome due to DGUOK deficiency is an extremely rare multiple mitochondrial DNA deletion syndrome with markedly decreased deoxyg uanosine kinase (DGUOK) activity in skeletal muscle characterized by a highly variable phenotype. Clinical manifestations include progressive external ophthalmoplegia, mitochondrial myopathy, recurrent rhabdomyolysis, lower motor neuron disease, mild cog nitive impairment, sensory axonal neuropathy, optic atrophy, ataxia, hypogonadism and/or parkinsonism. 